\title{
GENERALIZABLE KLEENE LOGICS
}

ATWELL R. TURQUETTE

In a recent paper [1], V. I. Šstakov distinguishes three calculi called the "regular Kleene calculus," the "Kleene calculus," and the "Kleene-Bočvar calculus." The regular Kleene calculus is based on a single binary operator $\nabla$, the Kleene calculus is based on two binary operators $\nabla$ and $\cong$, while the Kleene-Bočvar calculus is based on $\nabla$ and a single one-place operator $\vdash$. The truth-value properties of these operators are given by the following matrices:

\begin{tabular}{l|lll}
$\nabla$ & 1 & 2 & 3 \\
\hline 1 & 3 & 3 & 3 \\
2 & 3 & 2 & 2 \\
3 & 3 & 2 & 1
\end{tabular}

\begin{tabular}{l|lll}
$\cong$ & 1 & 2 & 3 \\
\hline 1 & 1 & 3 & 3 \\
2 & 3 & 1 & 3 \\
3 & 3 & 3 & 1
\end{tabular}

\begin{tabular}{l|l}
$P$ & $\vdash P$ \\
\hline 1 & 1 \\
2 & 3 \\
3 & 3
\end{tabular}

Sestakov's approach is very reasonable when one considers the fact that all of Kleene's basic operators [2] $-, \vee, \&, \rightarrow$, $\equiv$, and $\cong$ can be defined in terms of,$- V$, and $\cong$ alone. To see this, a standard 3-valued matrix or truth-table check can be used to justify the following definitions, where 1,2 , and 3 denote Kleene's $t, u$, and $f$ :

$$
\begin{aligned}
& P \& Q={ }_{\mathrm{d} f}-((-P) \vee(-Q)), \\
& P \rightarrow Q={ }_{\mathrm{d} f}(-P) \vee Q, \\
& P \equiv Q={ }_{\mathrm{d} f} P \rightarrow Q \& Q \rightarrow P .
\end{aligned}
$$

In like fashion, it is easy to show that Kleene's original strong logic $[2$, p. 336] is functionally equivalent to Sestakov's Kleene calculus in the sense that both calculi define the same set of truth-value functions. This follows immediately by the following definitions:

$$
\begin{gathered}
-P={ }_{\mathrm{d} f} P \nabla P, \\
P \vee Q={ }_{\mathrm{df}}-(P \nabla Q), \\
P \nabla Q={ }_{\mathrm{df}}-(P \vee Q) .
\end{gathered}
$$

Clearly, symmetry indicates that similar results can be obtained by introducing a binary operator $\Delta$ which is such that its associated matrix is the same as the matrix associated with $-(P \& Q)$.

Presented to the Society, November 25, 1966; received by the editors December 6, 1966. 
It is not difficult to show also that Šestakov's Kleene calculus, the Kleene-Bočvar calculus, and the 3-valued logic of Lukasiewicz based on the operators $\boldsymbol{C}$ and $\boldsymbol{N}$ [3] are all functionally equivalent. The following definitions are sufficient to establish the equivalence of Šestakov's Kleene calculus and the Kleene-Bočvar calculus:

$$
\begin{aligned}
\vdash P & ={ }_{\mathrm{d} f} P \cong(P \cong P), \\
J_{3} P & ={ }_{\mathrm{d} f} \vdash(-P), \\
J_{2} P & ={ }_{\mathrm{d} \mathfrak{}}(\vdash P) \nabla J_{3} P, \\
P C Q & ={ }_{\mathrm{d} f}-\left(J_{2} P \& J_{2} Q\right) \rightarrow(P \rightarrow Q), \\
P \cong Q & ={ }_{\mathrm{d} f} \vdash(P C Q) \&(Q C P) .
\end{aligned}
$$

Since Kleene's - corresponds to Lukasiewicz' $N$, the above definitions together with the following suffice to establish the functional equivalence of Šstakov's Kleene calculus, the Kleene-Bočvar calculus, Kleene's original strong calculus, and Łukasiewicz' calculus based on the 3-valued operators $C$ and $N$ :

$$
\begin{gathered}
P \nabla Q={ }_{\mathrm{df}} N((P C Q) C Q), \\
\vdash P={ }_{\mathrm{df}} N(P C N P) .
\end{gathered}
$$

It now seems natural to ask if Šestakov's approach can be generalized to the $M$-valued case for all finite $M$ greater than 3 . The results which follow will indicate that the answer to such a question is negative. To this end, let $h(p, q)$ denote the truth-value function associated with $P \nabla Q$. Let $j(p)$ denote the truth-value function associated with $\vdash P$, and let $e(p, q)$ denote the truth-value function associated with $P \cong Q$. A generalized $\nabla$ will be such that $h(p, q)=M-\min (p, q)+1$. A generalized $\vdash$ will be such that $j(p)=1$ if $p=1$ and $j(p)=M$ if $p \neq 1$. Likewise, a generalized $\cong$ will be such that $e(p, q)=1$ if $p=q$ and $e(p, q)=M$ if $p \neq q$. Using these generalized $\nabla, \vdash$, and $\cong$ the following theorem can be proved:

THEOREM. Let $W$ be a well-formed formula built up out of $P$ by means of the generalized operators $\nabla, \vdash$, and $\cong$. If $M>3$ and $P$ is assigned a value from the set $\{1,2, M-1, M\}$, then $W$ will take a value from the set $\{1,2, M-1, M\}$.

Proof. Use strong mathematical induction on the form or structure of $W$.

$(\alpha)$ If $W$ is $P$, the theorem is clear.

( $\beta$ ) Case 1. If $W$ is of the form $\vdash U$ or of the form $U_{1} \cong U_{2}$, then the theorem follows immediately from the fact that the truth-value 
functions $j$ and $e$ take only the values 1 and $M$.

Case 2. If $W$ is of the form $U_{1} \nabla U_{2}$, then by the assumption of induction the theorem holds for both $U_{1}$ and $U_{2}$. Hence, both $U_{1}$ and $U_{2}$ take values from the set $\{1,2, M-1, M\}$ when $P$ is assigned values from this set. Let $P$ be assigned values from the set $\{1,2$, $M-1, M\}$. By checking the value of the truth-value function $h$ for each of the sixteen ways in which $U_{1}$ and $U_{2}$ can take values from the set $\{1,2, M-1, M\}$, it is readily seen that $U_{1} \nabla U_{2}$ takes values which are restricted to this set. Hence, the theorem follows in this case.

Note that the theorem would not be altered if Słupecki's tertium operator $T$ [3] were used with those mentioned in the theorem to build up the well-formed formula $W$. This is clear since the truthvalue function associated with $T$ always takes the value 2 . Now let $M>4$ and consider a logic based on either the set of operators $\{\nabla, \vdash, T\}$ or $\{\nabla, \cong, T\}$. Such a logic could not define a constant function which always takes the value 3 and is, thus, functionally incomplete. On the other hand, it is known that a logic based on the set of operators $\{C, N, T\}$ is functionally complete for each $M$ [3]. Hence, if Sestakov's Kleene calculus and Kleene-Bočvar calculus are generalized to the $M$-valued case, they do not as in the 3-valued case always remain functionally equivalent to Eukasiewicz' logic based on the operators $C$ and $N$. In particular, for $M>4$, they are not functionally equivalent to the $M$-valued Lukasiewicz logic. Further, the reader will find it an interesting exercise to show that for $M=4$, the generalized Kleene calculus is functionally equivalent to the 4-valued Eukasiewicz logic but the latter is not functionally equivalent to the generalized 4-valued Kleene-Bocvar calculus.

Does a similar result follow from generalizing Kleene's original strong logic? The answer is affirmative if such logics are thought of as being based on the set of generalized operators $\{-, V, \cong\}$ or $\{-, \&, \cong\}$. The generalized version of Kleene's original strong logic would then be functionally equivalent to the generalized version of Sestakov's Kleene calculus. Contrariwise, the answer is negative if the generalized version of Kleene's original strong logic is thought of as based on the operators $\{\rightarrow,-, \cong\}$. It seems reasonable to use this set of operators, since it brings into focus the implication operator $\rightarrow$ and it is the difference between the truth-value properties of this operator and those of Eukasiewicz' implication operator which led Kleene [2] to distinguish his original strong logic from the 3valued Łukasiewicz calculus based on $C$ and $N$. Also, in the 3-valued case, Kleene's original strong logic, Sestakov's Kleene calculus, the 
Kleene-Bočvar calculus, Łukasiewicz' logic based on $\boldsymbol{C}$ and $\boldsymbol{N}$, and the logic based on the set of operators $\{\rightarrow,-, \cong\}$ are all functionally equivalent. This is an immediate consequence of the definitions which have already been introduced and the following:

$$
\begin{aligned}
& P \nabla Q==_{\mathrm{df}}-((-P) \rightarrow Q), \\
& P \rightarrow Q={ }_{\mathrm{df}}-((-P) \nabla Q) .
\end{aligned}
$$

In showing that a Kleene logic based on the set of operators $\{\rightarrow,-, \cong\}$ can be generalized in such a way that it will remain functionally equivalent to Lukasiewicz' $M$-valued logic based on $C$ and $N$, it will be convenient to use bracket-free notation. To this end, let $C^{\prime}$ denote an $M$-valued operator which is the generalized version of Kleene's implication operator $\rightarrow$. $C$ and $N$ will continue to be used for Eukasiewicz' implication and negation operators. Also, $\boldsymbol{N}$ will be used in place of Kleene's - and $E^{\prime}$ will be used in place of Kleene's $\cong$. Let $\boldsymbol{C} p q$ denote the truth-value function associated with $C P Q$, $C^{\prime} p q$ the truth-value function associated with $C^{\prime} P Q, E^{\prime} p q$ the truthvalue function associated with $E^{\prime} P Q$, and $N p$ the truth-value function associated with $N P$. The truth-value properties of the generalized $M$-valued operators $C, C^{\prime}, E^{\prime}$, and $N$ may now be expressed as follows:

$$
\begin{aligned}
C_{p q} & =\max (1, q-p+1) \\
C^{\prime} p q & =2 \text { if } p=q=2, \\
& =M-1 \quad \text { if } p=q=M-1, \\
& =C p q \text { in all other cases; } \\
E^{\prime} p q & =1 \quad \text { if } p=q, \\
& =M \quad \text { if } p \neq q ; \\
N_{p} & =M-p+1 .
\end{aligned}
$$

What is desired is to show that an $M$-valued logic based on $\left\{C^{\prime}, N, E^{\prime}\right\}$ is functionally equivalent to an $M$-valued logic based on $\{C, N\}$. Following Šstakov, a logic based on $\left\{C^{\prime}, N, E^{\prime}\right\}$ might be called a generalized or $M$-valued Kleene calculus. Hence, it is desired to show that the generalized $M$-valued Kleene calculus is functionally equivalent to the $M$-valued Lukasiewicz logic based on $C$ and $N$.

It will be shown first that the generalized $M$-valued Kleene calculus is functionally equivalent to an $M$-valued logic based on $C^{\prime}, N$, and a generalized Bočvar operator $\vdash[4]$. Let $J_{k}$ denote the general- 
ized version of Bočvar's operator. If $J_{k} p$ is the truth-value function associated with $J_{k} P$, then the truth-value properties of the generalized version of Bočvar's operator may be expressed as follows [3]:

$$
\begin{aligned}
J_{k} p & =1 \quad \text { if } p=k, \\
& =M \quad \text { if } p \neq k .
\end{aligned}
$$

Again following Sestakov, a logic based on the set of $M$-valued operators $\left\{C^{\prime}, N, J_{1}\right\}$ might be called a generalized or $M$-valued KleeneBočvar calculus. Hence, it is desired to show that the generalized Kleene calculus is functionally equivalent to the $M$-valued KleeneBočvar calculus. To this end consider the following definition:

$$
J_{1} P={ }_{\mathrm{df}} E^{\prime} P E^{\prime} P P .
$$

In view of the truth-value properties of $J_{1}$ and $E^{\prime}$, it is easy to check this definition and it follows that all the truth-value functions which can be defined in the $M$-valued Kleene-Bočvar calculus can also be defined in the generalized Kleene calculus. It remains to establish the converse. For the moment, assume that $J_{k} P$ can be defined in the $M$-valued Kleene-Bočvar logic. In this logic it will then be possible to define a binary operator $\boldsymbol{Z}_{k}$ which is such that its truthvalue properties may be described as follows when $\boldsymbol{Z}_{k} p q$ denotes the truth-value function associated with $Z_{k} P Q$ :

$$
\begin{aligned}
Z_{k} p q & =M \quad \text { if } p=q=k \\
& =1 \text { in all other cases. }
\end{aligned}
$$

Consider the following definition of $\boldsymbol{Z}_{\boldsymbol{k}}$ :

$$
Z_{k} P Q={ }_{\mathrm{d} f} C^{\prime} C^{\prime} N J_{k} P N J_{k} Q N J_{k} Q .
$$

To justify this definition, note that when $Q$ does not take the value $k$, $N J_{k} Q$ takes the value 1 and so does $\boldsymbol{Z}_{k} P Q$ by the truth-value properties of $\boldsymbol{C}^{\prime}$. If $Q$ does take the value $k, N J_{k} Q$ takes the value $M$. So if $P$ does not take the value $k, C^{\prime} N J_{k} P N J_{k} Q$ also takes the value $M$ and $Z_{k} P Q$ then takes the value 1 . On the other hand, if $P$ and $Q$ both take the value $k, C^{\prime} N J_{k} P N J_{k} Q$ takes the value 1 and both $N J_{k} Q$ and $Z_{k} P Q$ take the value $M$.

In order to show that all truth-value functions which can be defined in the generalized Kleene calculus can also be defined in the $M$ valued Kleene-Bočvar calculus, it is sufficient to define $E^{\prime}$ in the latter calculus. For this purpose, it is not necessary to define $\boldsymbol{Z}_{k}$ for all values of $k$. It is sufficient to define $Z_{2}$ and $Z_{M-1}$. Hence, from the above definition of $\boldsymbol{Z}_{k}$, it will only be necessary to define $J_{2}$ and $J_{M-1}$. These may be defined as follows: 


$$
\begin{aligned}
J_{2} P & ={ }_{\mathrm{d} t} N J_{1} C^{\prime} C^{\prime} P P C^{\prime} N P N P, \\
J_{M-1} P & ={ }_{\mathrm{df}} J_{2} N P .
\end{aligned}
$$

If the definition of $J_{2} P$ can be justified, it is easy to justify the definition of $J_{M-1} P$. To justify the definition of $J_{2} P$, note that when $C^{\prime} P P$ takes the value $2, C^{\prime} N P N P$ takes the value $N 2$ and vice versa. It is now possible to define Lukasiewicz' $\boldsymbol{C}$ as follows:

$$
C P Q={ }_{\mathrm{df}} \boldsymbol{C}^{\prime} \boldsymbol{Z}_{2} P Q \boldsymbol{C}^{\prime} \boldsymbol{Z}_{M-1} P Q \boldsymbol{C}^{\prime} P Q .
$$

This definition is easily justified making use of the truth-valued properties of $\boldsymbol{C}^{\prime}$ and $\boldsymbol{Z}_{k}$. With $\boldsymbol{C}$ defined, the following rather standard definitions can be used to define $E^{\prime}$ :

$$
\begin{aligned}
& A P Q==_{\mathrm{d} f} C C P Q Q, \\
& K P Q={ }_{\mathrm{df}} N A N P N Q, \\
& E^{\prime} P Q=\mathrm{df}_{\mathrm{df}} K C P Q C Q P .
\end{aligned}
$$

It is now possible to conclude that the generalized Kleene calculus is functionally equivalent to the $M$-valued Kleene-Bočvar calculus.

To show that the generalized Kleene logic is functionally equivalent to Eukasiewicz' $M$-valued logic based on $C$ and $N$, it will be shown that the generalized Kleene-Bočvar logic is functionally equivalent to the $M$-valued logic of Lukasiewicz based on the operators $\boldsymbol{C}$ and $\boldsymbol{N}$. From the above definition of $\boldsymbol{C}$, it is clear that all the truth-value functions which can be defined in Lukasiewicz' $M$-valued logic based on $\boldsymbol{C}$ and $\boldsymbol{N}$ can also be defined in the generalized Kleene-Bočvar logic. Hence, it is only necessary to consider the converse. For this, it is sufficient to define $J_{1}$ and $C^{\prime}$ in terms of $C$ and $N$ alone. It is known that for each $k, J_{k}$ can be defined in terms of $C$ and $N$ alone [3] and the above definitions make it clear that $K$ can be defined in terms of $\boldsymbol{C}$ and $\boldsymbol{N}$ alone. The following definitions can thus be used to define $\boldsymbol{C}^{\prime}$ in terms of $\boldsymbol{C}$ and $\boldsymbol{N}$ alone:

$$
\begin{aligned}
& U P Q==_{\mathrm{d} f} C N K J_{2} P J_{2} Q C N K J_{M-1} P J_{M-1} Q C P Q, \\
& V P Q={ }_{\mathrm{d} f} C K J_{2} P J_{2} Q P, \\
& W P Q={ }_{\mathrm{df}} C K J_{M-1} P J_{M-1} Q P, \\
& C^{\prime} P Q={ }_{\mathrm{d} \mathrm{f}} K K U P Q V P Q W P Q .
\end{aligned}
$$

This definition of $\boldsymbol{C}^{\prime}$ may seem complex, but it is highly intuitive in the light of the truth-value properties of $C^{\prime}$. In particular, note that $K$ is a maximum operator and that essentially $U P Q$ gives the values of $C^{\prime} P Q$ when $P$ and $Q$ are neither both 2 nor both $M-1$. On the 
other hand, $V P Q$ gives the value of $C^{\prime} P Q$ when $P$ and $Q$ are both 2 . Likewise, $W P Q$ gives the value of $C^{\prime} P Q$ when $P$ and $Q$ both take the value $M-1$.

From this definition of $\boldsymbol{C}^{\prime}$ and the results already established, it follows that the generalized Kleene logic based on $\left\{C^{\prime}, N, E^{\prime}\right\}$ is functionally equivalent to Łukasiewicz' $M$-valued logic based on $\{C, N\}$. In fact, it is now clear that $M$-valued logics based on the sets $\left\{C^{\prime}, N, E^{\prime}\right\},\left\{C^{\prime}, N, J_{1}\right\}$, and $\{C, N\}$ are all functionally equivalent. This is the desired result and it follows at once that, although Šestakov's operator $\nabla$ can not be generalized to yield logics which are functionally equivalent to Łukasiewicz' $M$-valued logics based on $C$ and $N$, it is possible to generalize Kleene's implication operator $\rightarrow$ and obtain logics which remain functionally equivalent to Eukasiewicz' logics based on the $M$-valued operators $C$ and $N$.

\section{REFERENCES}

1. V. I. Sestakov, On the relationship between certain three-valued logical calculi, Uspehi Mat. Nauk 19 (1964), no. 2 (116), 177-181. Also see Math. Reviews 31 (1966), $\# 2140$.

2. Stephen C. Kleene, Introduction to metamathematics, Van Nostrand, Princeton, N. J., 1952; pp. 332-337.

3. J. B. Rosser and A. R. Turquette, Many-valued logics, North-Holland, Amsterdam, 1952; Chapter II.

4. See Alonzo Church's review of Bočvar in J. Symbolic Logic 4 (1939), 98-99.

UNIVERSITY OF ILLINOIS 